\title{
Influence of Poultry Manure Rates and Spacing on Growth, Yield, Nutrient Concentration, Uptake and Proximate Composition of Onion (Allium cepa L.)
}

\author{
Ehizogie J. FALODUN ${ }^{1 *}$, Racheal K.A. EGHAREVBA ${ }^{2}$ \\ ${ }^{1}$ University of Benin, Faculty of Agriculture, Department of Crop Science, Nigeria; ehizogie.falodun@uniben.edu (*corresponding author) \\ ${ }^{2}$ University of Benin, Faculty of Agriculture, Department of Crop Science, Nigeria; ranoiteg@yahoo.com
}

\begin{abstract}
Plant spacing determines to a greater extent crop performance in terms of growth and yield. The production of crop with organic fertilizer also plays a vital role in organic agriculture. Field studies were conducted to evaluate the effects of spacing and poultry manure on the growth, yield and quality of onion. Three spacing regimes were carried out consisting of $15 \mathrm{~cm} \times 15 \mathrm{~cm}$, $20 \mathrm{~cm} \times 20 \mathrm{~cm}$ and $25 \mathrm{~cm} \times 25 \mathrm{~cm}$ and four levels of poultry manure at $0,5,10$ and $15 \mathrm{t} / \mathrm{ha}$. The effects of spacing and poultry manure were evaluated for two years based on plant growth, yield, nutrient concentration, uptake and proximate composition of onion plant. Leaf thickness, bulb and shoot fresh weights were significantly increased by the wider spacing of $20 \mathrm{~cm} \times 20 \mathrm{~cm}$ and $25 \mathrm{~cm} \times 25 \mathrm{~cm}$, compared with the narrower spacing of $15 \mathrm{~cm} \times 15 \mathrm{~cm}$ in both seasons. However, the highest total dry yield (1.82 and 1.58) t/ha, shoot yield (2.31 and 1.32) t/ha and total fresh yield (13.69 and 12.55) t/ha were obtained with the spacing of $20 \mathrm{~cm} \times 20 \mathrm{~cm}$ in both years. Similarly, application of poultry manure increased leaf thickness, bulb and shoot fresh weights and yields compared with the control. Generally, using $10 \mathrm{t} /$ ha poultry manure has a superior effect on proximate composition and most of growth parameters and yield components achieved the highest nutrient concentrations and uptake on most of the macro and micronutrients in leaves and bulbs as compared with the control in both years.
\end{abstract}

Keywords: bulb; control; leaf thickness; shoot; quality

\section{Introduction}

Worldwide, onion (Allium cepa $\mathrm{L}$.) is a vegetable crop of commercial importance and has been the most used flavouring vegetable for centuries. According to Hussaini et al. (2000), the crop ranks second in importance after tomatoes among the vegetables in Nigeria. Aliyu et al. (2008) documented that it is grown mainly for its bulbs, which are used almost daily in every home in Nigeria. It is ranked among the top 5\% of most important vegetables in Nigeria based on level of consumption and other uses (NIHORT, 1986). Plant spacing and manure application are important agronomic factors which can cause substantial increase or decrease in yield of most crops hence, appropriate plant spacing is vital for the interception of enough sunlight necessary for optimum photosynthesis (Aliyu et al., 2008). Proper spacing ensures optimum plant growth through adequate utilization of moisture, light and nutrients. Baloch et al. (2001) observed that increasing plant spacing increased the nutrient area per plant and this led to increase in morphological characters. Mortley et al. (1992) noted that stem diameter increased linearly as spacing between plants increased and that fresh weight yields are highest at closer within-row spacing. Pandey et al. (1996) observed the highest plant height in narrow spacing in tomato hybrids, while wider spacing had the highest number of primary branches per plant. They attributed the higher plant height recorded in narrow spacing to greater competition for space and light and thereby forcing the plants to grow taller. In many developing countries, farmers have limited financial resources and can rarely afford to purchase sufficient mineral fertilizers at the same time. The problem of affordability and procurement of chemical fertilizers by resource-poor farmers make the use of poultry manures a viable alternative (Chen and Hammond, 1988). William et al. (1991) recommended organic manure application to cabbage production in the tropics based on the fact that organic manure improves soil water availability through retention, aeration and better response of crops to 
118

soil amendment materials. Organic manure alone or in combination with mineral fertilizer exerts more beneficial effect on fruit yield when compared to fertilizer applied alone (Ehigiator, 1998). Similarly, according to Adekiya and Agbede (2009) all levels of poultry manure alone and NPK fertilizer + poultry manure increased leaf $\mathrm{N}, \mathrm{P}, \mathrm{K}, \mathrm{Ca}$ and $\mathrm{Mg}$ amounts significantly, the concentration of nutrients increased with the amount of poultry manure up to $40 \mathrm{t}$ ha NPK fertilizer + poultry manure gave higher leaf $\mathrm{N}, \mathrm{P}$ and $\mathrm{K}$ contents than poultry manure alone. According to Aisha et al. (2007), crops cultivated with organic manures are not only free from harmful chemicals, they are also safer, healthier and tastier. Apart from supplying plant nutrients, Akinfasoye and Akanbi (2005) also justified that they improve soil physical and microbial properties and eliminate pollution of underground water. Onions are more susceptible than other crops in extracting nutrients, especially the immobile types such as $\mathrm{Ca}, \mathrm{Mn}, \mathrm{Fe}$ and $\mathrm{Zn}$, because of their shallow and unbranched root system; hence they require and often respond well to addition of fertilizers (Brewester, 1994). Onion is a heavy feeder, requiring ample supplies of nutrients. The use of organic manure and compost has been shown to improve the soil organic matter content and crop yield (Soumare et al., 2003). The mineral composition of crops depends on the amount and type of nutrients taken from the growth medium, and it is necessary that adequate amount of nutrients should be available for the production and nutrient content of crops (Barker et al., 2006). The organic manure is very important in improving soil productivity and its function is performed through the improvement of the physical condition of the soil structure (Lombin et al., 1991). Adenawoola and Adejoro (2005) also found out that organic matter and soil nutrients increased with application rate of poultry manure and therefore affirmed that poultry manure contains organic matter, $\mathrm{N}, \mathrm{P}$, $\mathrm{K}, \mathrm{Ca}$ and $\mathrm{Mg}$ which are released into the soil upon decomposition of the manure, and that depletion of soil organic matter under intensive cropping can be amended by proper addition of poultry manure into the soil.

In this light, the present study was carried out to determine the effect of spacing and poultry manure on the growth, yield, nutrient concentration and uptake of onion plant.

\section{Materials and Methods}

\section{Soilproperties}

The study was carried out in the 2011/2012 and 2012/2013 dry seasons at the Teaching and Research Farm of the University of Benin, Ugbowo Campus, Benin-City, in Edo State, Nigeria. The climate was tropical and the vegetation was lowland rainforest in the south (with mean annual rainfall of $2300 \mathrm{~mm}$ ) to guinea savanna in Edo North with 1,400 mm mean rainfall. Prior to analysis, the soil samples were air dried and crushed to pass through a $2 \mathrm{~mm}$ sieve. Soil pH was determined using a $\mathrm{pH}$ meter. Organic carbon was determined by Walkley and Black (1962) wet oxidation method as modified by Jackson (1969). Total nitrogen was obtained by macro Kjeldahl methods as modified by Jackson (1969). Available P was extracted by Bray I method (Bray and Kurtz, 1945) and the P was estimated by the blue colour method of Murphy and Riley (1962).
Exchangeable $\mathrm{K}$ and $\mathrm{Na}$ were determined using flame photometer, and $\mathrm{Ca}$ and $\mathrm{Mg}$ using the Atomic Absorption Spectrophotometer (Table 1).

\section{The study design}

The experiment was conducted as a randomized complete block design (RCBD) with three replications. The treatments were composed of a factorial combinations of four rates $(0,5,10$ and $15 \mathrm{t} /$ ha poultry manure) and three spacing $15 \times 20,20 \times 20$ and $25 \times 25 \mathrm{~cm}$. Each replicate had 12 plots giving a total of 36 plots in this experiment.

The onion seeds of 'Kano red', a local cultivar, were sown in the nursery and transplanted to the field when seedlings were seven weeks after sowing and at about $14 \mathrm{~cm}$ tall. Plots were mulched to conserve soil moisture and suppress weeds. Data collection started four weeks after transplanting. Four plants were randomly selected from each plot and tagged for the purpose of collecting data for leaf length, individual bulb and shoot weight, leaf thickness, harvest index, bulb yield and total fresh yield per hectare.

Determination of nutrient concentration and uptake of onion bulbs

At $101 \mathrm{DAT}$ (at harvest) bulbs were harvested, cleaned of soil particles and weighed with a sensitive balance. The bulbs were chopped into small bits and oven dried in a forced-Air Sanyo Gallen kamp moisture extraction oven at $70{ }^{\circ} \mathrm{C}$ to a constant weight (ISTA, 1993). The dried samples were there after weighed using a sensitive electronic balance and the weight recorded. These were milled to pass through $0.2 \mathrm{~mm}$ sieve using Thomas Wiley Hammer Mill in preparation for laboratory analysis. Calcium (Ca), Sodium $(\mathrm{Na})$ and Magnesium $(\mathrm{Mg})$ along with $\mathrm{N}, \mathrm{P}$ and $\mathrm{K}$ were determined following standard laboratory procedures. The analytical procedures for the nutrients were as described by AOAC (1990). Nitrogen, P, K, Ca, Na and $\mathrm{Mg}$ uptake were derived from multiplying the oven dry weight of the tissue with the corresponding value of the nutrient in the tissue.

Table 1. Physical and chemical properties of poultry manure and soil of the experimental site pre plant and post-harvest

\begin{tabular}{|c|c|c|c|}
\hline \multirow{2}{*}{ Soil properties } & \multirow{2}{*}{$\begin{array}{l}\text { Poultry } \\
\text { manure }\end{array}$} & \multicolumn{2}{|c|}{ Experimental soil } \\
\hline & & Pre & Post \\
\hline $\mathrm{pH}\left(\mathrm{H}_{2} \mathrm{O}\right)$ & 6.40 & 4.80 & 5.26 \\
\hline Organic carbon $\left(\mathrm{g}^{100 \mathrm{~g}^{-1}}\right)$ & 23.00 & 0.40 & 0.73 \\
\hline Total $\mathrm{N}\left(\mathrm{g} 100 \mathrm{~g}^{-1}\right)$ & 2.13 & 0.05 & 0.03 \\
\hline Total P $\left(\mathrm{mg} \mathrm{kg}^{-1}\right)$ & 4.30 & 18.9 & 14.7 \\
\hline $\mathrm{K}\left(\mathrm{cmol} \mathrm{kg}^{-1}\right)$ & 1.12 & 0.19 & 0.12 \\
\hline $\mathrm{Ca}\left(\mathrm{cmol} \mathrm{kg}^{-1}\right)$ & 3.76 & 1.15 & 0.81 \\
\hline $\mathrm{Mg}\left(\mathrm{cmol} \mathrm{kg}^{-1}\right)$ & N/A & 0.74 & 0.56 \\
\hline $\operatorname{Mn}\left(\mathrm{cmol} \mathrm{kg}^{-1}\right)$ & 1.14 & $\mathrm{~N} / \mathrm{A}$ & $\mathrm{N} / \mathrm{A}$ \\
\hline $\mathrm{Zn}\left(\mathrm{cmol} \mathrm{kg}^{-1}\right)$ & 0.13 & $\mathrm{~N} / \mathrm{A}$ & $\mathrm{N} / \mathrm{A}$ \\
\hline $\mathrm{Fe}\left(\mathrm{cmol} \mathrm{kg}^{-1}\right)$ & 3.27 & $\mathrm{~N} / \mathrm{A}$ & $\mathrm{N} / \mathrm{A}$ \\
\hline Sand $\left(\mathrm{g} \mathrm{kg}^{-1}\right)$ & N/A & 752.1 & 756.0 \\
\hline Clay $\left(\mathrm{g} \mathrm{kg}^{-1}\right)$ & $\mathrm{N} / \mathrm{A}$ & 198.0 & 163.4 \\
\hline Silt $\left(\mathrm{g} \mathrm{kg}^{-1}\right)$ & $\mathrm{N} / \mathrm{A}$ & 46.0 & 48.2 \\
\hline Textural class & $\mathrm{N} / \mathrm{A}$ & \multicolumn{2}{|c|}{ Sandy loam } \\
\hline
\end{tabular}

N/A - Not available 


\section{Statistical analysis}

The data obtained were subjected to statistical analysis of variance (ANOVA) using SAS following the model for factorial experiment in a randomized complete block design and means separated by Duncan Multiple Range Test (DMRT).

\section{Results}

In both years, the effect of spacing and poultry manure on crop growth rate and leaf thickness of onion was significant. Wider spacing of $20 \mathrm{~cm} \times 20 \mathrm{~cm}$ and $25 \mathrm{~cm} \times$ $25 \mathrm{~cm}$ significantly increased crop growth rate and leaf thickness above the narrower spacing of $15 \mathrm{~cm} \times 20 \mathrm{~cm}$. However, the effect of spacing on harvest intervals in both years was not significant. The shoot fresh weight per plant increased with increase in spacing with the highest shoot fresh weight produced at $25 \mathrm{~cm} \times 25 \mathrm{~cm}$ spacing in both years. Similarly, the effect of poultry manure was significant for all the parameters measured except for harvest interval in 2010 cropping season which was not significant. In all cases plants treated with either 10 or $15 \mathrm{t} \mathrm{ha}^{-1}$ poultry manure produced similar results and these were significantly increased above the other treatments (Table 2). The application of poultry manure increased crop growth rate and leaf thickness up to $10 \mathrm{t} / \mathrm{ha}$ and a further increase in application rate did not result in a significant increase in these parameters. However, shoot fresh weight per plant increased with increase in application rate up to $15 \mathrm{t} \mathrm{ha}^{-1}$. The effect of spacing on bulb fresh weight per plant was not significant in 2010/2011 cropping season however, in $2011 / 2012$ it was significant, narrower spacing of $15 \mathrm{~cm} \times$ $20 \mathrm{~cm}$ significantly produced lower bulb weight than wider spacing of $25 \mathrm{~cm} \times 25 \mathrm{~cm}$. In contrast to the bulb weight per plant, the highest shoot fresh yield and total dry yield was obtained at the narrower spacing of either $15 \mathrm{~cm} \times 20$ $\mathrm{cm}$ or $20 \mathrm{~cm} \times 20 \mathrm{~cm}$. The effect of manure application was significant on shoot fresh, dry and total dry yield and these increased with increase in manure application up to $10 \mathrm{t} \mathrm{ha}^{-1}$ with the control producing the lowest yield for these parameters (Table 3). The nutrient concentration of $\mathrm{K}$ in onion leaves increased with increase in spacing however not significant in onion bulbs. The effect of poultry manure was significant in the concentration of $\mathrm{N}$ and $\mathrm{K}$ in both leaves and bulbs and significantly increased with increase in manure rates. However, there was no significant response in $\mathrm{P}$ content to increase in manure rates. The control significantly had the lowest concentrations of these nutrients (Tables 4 and 5).

The uptake of $\mathrm{N}, \mathrm{K}, \mathrm{Ca}$ and $\mathrm{Mg}$ decreased with increase in spacing in onion leaves and bulbs and then increased as the manure rates increased from $0 \mathrm{t} \mathrm{ha} \mathrm{h}^{-1}$ to $10 \mathrm{t} \mathrm{ha}^{-1}$. Nutrient concentration and uptake of $\mathrm{P}$ was not significant for both spacing and poultry manure application rates on onion leaves and bulbs. The effect of spacing on the proximate composition of onion leaves and bulbs were not significant however, the effect of manure application was significant for moisture content, ash and percentage dry matter content of onion leaves. The moisture content increased with increase in manure rates up to $10 \mathrm{t} \mathrm{ha}^{-1}$ while the ash content increased up to $5 \mathrm{tha}^{-1}$. There was an inverse relationship observed with the percentage dry matter content of onion leaves and bulbs when compared with the percentage moisture content (Tables 8 and 9). The percentage of dry matter content decreased with increase in manure application rate lower application rates of 0 tha $^{-1}$ and $5 \mathrm{tha} \mathrm{a}^{-1}$ produced significantly higher percentage of dry matter content above the higher application rate of $15 \mathrm{t} \mathrm{ha}^{-1}$, while the percentage moisture content increased with increase in manure application from $0-15 \mathrm{t} \mathrm{ha}^{-1}$ (Tables 8 and 9).

Table 2. Effect of spacing and poultry manure on crop growth rate (CGR) $\mathrm{gm}^{-2} \mathrm{wk}^{-1}$, leaf thickness $(\mathrm{cm})$, harvest index and shoot fresh wt/plant (g) of onion 2010/2011 and 2011/2012 dry cropping seasons

\begin{tabular}{|c|c|c|c|c|c|c|c|c|}
\hline \multirow[b]{2}{*}{ Treatment } & \multicolumn{5}{|c|}{ 2010/2011 Cropping season } & \multicolumn{3}{|c|}{ 2011/2012 Cropping season } \\
\hline & $\begin{array}{c}(\text { CGR }) \\
\left(\mathrm{gm}^{2} \mathrm{wk}^{-1}\right)\end{array}$ & $\begin{array}{c}\text { Leaf } \\
\text { thickness }(\mathrm{cm})\end{array}$ & $\begin{array}{l}\text { Harvest } \\
\text { Index }\end{array}$ & $\begin{array}{c}\text { Shoot } \\
\text { fresh wt/plant } \\
(\mathrm{g})\end{array}$ & $\begin{array}{c}(\mathrm{CGR}) \\
\left(\mathrm{gm}^{-2} \mathrm{wk}^{-1}\right)\end{array}$ & $\begin{array}{c}\text { Leaf } \\
\text { thickness }(\mathrm{cm})\end{array}$ & $\begin{array}{l}\text { Harvest } \\
\text { Index }\end{array}$ & $\begin{array}{c}\text { Shoot } \\
\text { fresh wt/plant } \\
(\mathrm{g})\end{array}$ \\
\hline \multicolumn{9}{|l|}{ Spacing $(\mathrm{cm})$} \\
\hline $15 \times 20$ & $0.39^{\mathrm{b}}$ & $0.09^{\mathrm{b}}$ & $0.65^{\mathrm{b}}$ & $6.89^{\mathrm{b}}$ & $0.37^{\mathrm{b}}$ & $0.07^{\mathrm{b}}$ & $0.87^{\mathrm{a}}$ & $3.95^{\mathrm{b}}$ \\
\hline $20 \times 20$ & $0.42^{\mathrm{ab}}$ & $0.13^{a}$ & $0.66^{\mathrm{ab}}$ & $7.04^{b}$ & $0.39^{a}$ & $0.11^{\mathrm{a}}$ & $0.88^{\mathrm{a}}$ & $5.31^{a}$ \\
\hline $25 \times 25$ & $0.43^{a}$ & $0.14^{a}$ & $0.69^{\mathrm{a}}$ & $9.25^{a}$ & $0.40^{2}$ & $0.12^{\mathrm{a}}$ & $0.88^{\mathrm{a}}$ & $5.65^{a}$ \\
\hline SEM & 0.01 & 0.01 & 0.03 & 1.22 & 0.01 & 0.01 & 0.04 & 1.10 \\
\hline \multicolumn{9}{|c|}{$\begin{array}{l}\text { Poultry manure } \\
\qquad\left(\mathrm{th} \mathrm{h}^{-1}\right)\end{array}$} \\
\hline 0 & $0.32^{\mathrm{b}}$ & $0.03^{c}$ & $0.59^{\mathrm{b}}$ & $5.25^{\mathrm{b}}$ & $0.19^{c}$ & $0.02^{\mathrm{b}}$ & $0.84^{\mathrm{a}}$ & $2.48^{\mathrm{d}}$ \\
\hline 5 & $0.34^{b}$ & $0.13^{\mathrm{ab}}$ & $0.67^{\mathrm{ab}}$ & $6.37^{\mathrm{ab}}$ & $0.30^{\mathrm{b}}$ & $0.12^{\mathrm{a}}$ & $0.86^{\mathrm{a}}$ & $4.75^{c}$ \\
\hline 10 & $0.42^{\mathrm{a}}$ & $0.14^{\mathrm{a}}$ & $0.72^{\mathrm{a}}$ & $8.72^{\mathrm{ab}}$ & $0.40^{2}$ & $0.13^{\mathrm{a}}$ & $0.88^{\mathrm{a}}$ & $5.30^{b}$ \\
\hline 15 & $0.43^{\mathrm{a}}$ & $0.16^{a}$ & $0.72^{\mathrm{a}}$ & $10.36^{a}$ & $0.42^{\mathrm{a}}$ & $0.13^{a}$ & $0.91^{a}$ & $7.37^{\mathrm{a}}$ \\
\hline SEM & 0.02 & 0.03 & 0.10 & 1.67 & 0.03 & 0.02 & 0.12 & 0.13 \\
\hline
\end{tabular}

Means followed by the same letter in a column are not significantly different at $5 \%$ level of probability using DMRT. 
Table 3. Effect of poultry manure and spacing on yield and yield components of onion 2010/2011 and 2011/2012 dry cropping seasons

\begin{tabular}{|c|c|c|c|c|c|c|c|c|}
\hline \multirow[b]{2}{*}{ Treatment } & \multicolumn{4}{|c|}{ 2010/2011 Cropping season } & \multicolumn{3}{|c|}{ 2011/2012 Cropping season } & \multirow[b]{2}{*}{$\begin{array}{c}\text { Total dry yield } \\
\qquad\left(\mathrm{tha} \mathrm{a}^{-1}\right)\end{array}$} \\
\hline & $\begin{array}{c}\text { Bulb } \\
\text { fresh wt/bulb } \\
\text { (g) }\end{array}$ & $\begin{array}{c}\text { Shoot } \\
\text { fresh yield } \\
\left(\mathrm{tha}^{-1}\right)\end{array}$ & $\begin{array}{c}\text { Total fresh } \\
\text { yield } \\
\left(\mathrm{tha}^{-1}\right)\end{array}$ & $\begin{array}{c}\text { Total dry } \\
\text { yield }\left(\mathrm{tha}^{-1}\right)\end{array}$ & $\begin{array}{c}\text { Bulb } \\
\text { fresh wt/bulb } \\
\text { (g) }\end{array}$ & $\begin{array}{c}\text { Shoot } \\
\text { fresh yield } \\
(\mathrm{t} \mathrm{ha-1})\end{array}$ & $\begin{array}{c}\text { Total fresh } \\
\text { yield } \\
\left(\mathrm{t} \mathrm{ha}^{-1}\right)\end{array}$ & \\
\hline \multicolumn{9}{|l|}{ Spacing $(\mathrm{cm})$} \\
\hline $15 \times 20$ & 28.21 & $2.27^{\mathrm{a}}$ & $13.58^{\mathrm{a}}$ & $1.82^{\mathrm{a}}$ & $27.26^{\mathrm{b}}$ & $1.29^{\mathrm{b}}$ & $12.28^{\mathrm{a}}$ & $1.58^{\mathrm{a}}$ \\
\hline $20 \times 20$ & 34.92 & $2.31^{a}$ & $13.69^{\mathrm{a}}$ & $1.86^{\mathrm{a}}$ & $37.50^{\mathrm{ab}}$ & $1.32^{\mathrm{a}}$ & $12.69^{a}$ & $1.62^{\mathrm{a}}$ \\
\hline $25 \times 25$ & 39.87 & $1.13^{\mathrm{b}}$ & $9.76^{\mathrm{b}}$ & 0.94 & $40.45^{\mathrm{a}}$ & $0.90^{c}$ & $9.17^{\mathrm{b}}$ & $0.91^{\mathrm{b}}$ \\
\hline SEM & 10.02 & 0.23 & 1.04 & 0.12 & 10.24 & 0.04 & 1.02 & 0.20 \\
\hline \multicolumn{9}{|c|}{$\begin{array}{l}\text { Poultry manure } \\
\qquad\left(\text { tha }^{-1}\right)\end{array}$} \\
\hline 0 & $16.82^{\mathrm{c}}$ & $1.31^{\mathrm{b}}$ & $6.47^{\mathrm{c}}$ & $0.68^{\mathrm{c}}$ & $15.12^{\mathrm{c}}$ & $0.58^{\mathrm{d}}$ & $5.71^{\mathrm{c}}$ & $0.53^{\mathrm{c}}$ \\
\hline 5 & $30.32^{\mathrm{b}}$ & $1.58^{\mathrm{b}}$ & $10.03^{\mathrm{b}}$ & $1.34^{b}$ & $28.04^{\mathrm{b}}$ & $0.97^{\mathrm{c}}$ & $9.17^{\mathrm{b}}$ & $1.05^{\mathrm{b}}$ \\
\hline 10 & $42.78^{a}$ & $2.16^{\mathrm{ab}}$ & $14.01^{2}$ & $1.89^{\mathrm{a}}$ & $42.39^{\mathrm{a}}$ & $1.27^{\mathrm{b}}$ & $13.04^{\mathrm{a}}$ & $1.76^{\mathrm{a}}$ \\
\hline 15 & $45.42^{\mathrm{a}}$ & $2.56^{\mathrm{a}}$ & $15.34^{\mathrm{a}}$ & $2.27^{\mathrm{a}}$ & $44.63^{\mathrm{a}}$ & $1.74^{a}$ & $14.39^{\mathrm{a}}$ & $1.98^{\mathrm{a}}$ \\
\hline SEM & 10.02 & 0.25 & 1.07 & 0.15 & 10.24 & 0.05 & 1.08 & 0.22 \\
\hline
\end{tabular}

Means followed by the same letter in a column are not significantly different at $5 \%$ level of probability using DMRT.

Table 4. Effect of poultry manure and spacing on nutrient concentration of onion leaves

\begin{tabular}{|c|c|c|c|c|c|}
\hline \multirow{2}{*}{ Spacing $(\mathrm{cm})$} & \multicolumn{4}{|c|}{ Nutrient concentration } & \multirow[b]{2}{*}{$\mathrm{Mggkg}^{-1}$} \\
\hline & $\mathrm{Ng} \mathrm{kg}^{-1}$ & $\mathrm{Pg} \mathrm{kg}^{-1}$ & $\mathrm{Kg} \mathrm{kg}^{-1}$ & $\mathrm{Cagkg}^{-1}$ & \\
\hline $15 \times 20$ & 0.51 & 0.02 & $0.48^{\mathrm{b}}$ & 0.10 & 0.05 \\
\hline $20 \times 20$ & 0.52 & 0.02 & $0.51^{a}$ & 0.12 & 0.05 \\
\hline $25 \times 25$ & 0.53 & 0.03 & $0.51^{2}$ & 0.12 & 0.06 \\
\hline Significance & N.S & N.S & * & N.S & N.S \\
\hline SEM & 0.02 & 0.01 & 0.02 & 0.02 & 0.01 \\
\hline \multicolumn{6}{|c|}{ Poultry manure ( $\left.\mathrm{t} \mathrm{ha}^{-1}\right)$} \\
\hline 0 & $0.46^{c}$ & 0.01 & $0.30^{\mathrm{b}}$ & 0.08 & 0.05 \\
\hline 5 & $0.50^{\mathrm{b}}$ & 0.01 & $0.50^{\mathrm{a}}$ & 0.10 & 0.06 \\
\hline 10 & $0.54^{a}$ & 0.02 & $0.52^{\mathrm{a}}$ & 0.12 & 0.07 \\
\hline 15 & $0.56^{\mathrm{a}}$ & 0.02 & $0.52^{a}$ & 0.12 & 0.07 \\
\hline Significance & * & N.S & * & N.S & N.S \\
\hline SEM & 0.02 & 0.01 & 0.02 & 0.02 & 0.03 \\
\hline $\mathrm{P}^{*} \mathrm{~S}$ & N.S & N.S & N.S & N.S & N.S \\
\hline
\end{tabular}

Means followed by the same letter in a column are not significantly different at $5 \%$ level of probability.

${ }^{*}$ Significant at $5 \%$ level of probability.

Table 5. Effect of poultry manure and spacing on nutrient concentration of onion bulbs

\begin{tabular}{|c|c|c|c|c|c|}
\hline \multirow{2}{*}{ Spacing $(\mathrm{cm})$} & \multicolumn{4}{|c|}{ Nutrient concentration } & \multirow[b]{2}{*}{$\mathrm{Mgg} \mathrm{kg}^{-1}$} \\
\hline & $\mathrm{Ng} \mathrm{kg}^{-1}$ & $\mathrm{Pg} \mathrm{kg}^{-1}$ & $\mathrm{Kg} \mathrm{kg}^{-1}$ & $\mathrm{Cag} \mathrm{kg}^{-1}$ & \\
\hline $15 \times 20$ & 0.50 & 0.04 & 0.48 & 0.11 & 0.04 \\
\hline $20 \times 20$ & 0.52 & 0.05 & 0.48 & 0.12 & 0.05 \\
\hline $25 \times 25$ & 0.52 & 0.05 & 0.50 & 0.12 & 0.05 \\
\hline Significance & N.S & N.S & N.S & N.S & N.S \\
\hline SEM & 0.02 & 0.01 & 0.02 & 0.02 & 0.02 \\
\hline \multicolumn{6}{|c|}{ Poultry manure $\left(\mathrm{th}^{-1}\right)$} \\
\hline 0 & $0.43^{c}$ & 0.02 & $0.40^{\mathrm{b}}$ & $0.09^{\mathrm{b}}$ & 0.04 \\
\hline 5 & $0.47^{\mathrm{b}}$ & 0.05 & $0.42^{b}$ & $0.10^{\mathrm{ab}}$ & 0.05 \\
\hline 10 & $0.50^{\mathrm{a}}$ & 0.05 & $0.48^{\mathrm{a}}$ & $0.12^{\mathrm{a}}$ & 0.05 \\
\hline 15 & $0.52^{\mathrm{a}}$ & 0.05 & $0.50^{\mathrm{a}}$ & $0.12^{\mathrm{a}}$ & 0.06 \\
\hline Significance & ${ }^{*}$ & N.S & ${ }^{*}$ & ${ }^{*}$ & N.S \\
\hline SEM & 0.02 & 0.01 & 0.02 & 0.02 & 0.02 \\
\hline $\mathrm{P}^{*} \mathrm{~S}$ & N.S & N.S & N.S & N.S & N.S \\
\hline
\end{tabular}

Means followed by the same letter in a column are not significantly different at $5 \%$ level of probability.

${ }^{*}$ Significant at $5 \%$ level of probability. 
Table 6. Effect of poultry manure and spacing on nutrient uptake of onion leaves

\begin{tabular}{|c|c|c|c|c|c|}
\hline \multirow{2}{*}{$\begin{array}{c}\text { Spacing } \\
(\mathrm{cm})\end{array}$} & \multicolumn{4}{|c|}{ Nutrient uptake $\left(\mathrm{kg} \mathrm{ha}^{-1}\right)$} & \multirow[b]{2}{*}{$\mathrm{Mg}$} \\
\hline & $\mathrm{N}$ & $\mathrm{P}$ & $\mathrm{K}$ & $\mathrm{Ca}$ & \\
\hline $15 \times 20$ & $36.68^{a}$ & 1.36 & $34.49^{a}$ & $6.49^{a}$ & $3.55^{\mathrm{ab}}$ \\
\hline $20 \times 20$ & $43.42^{\mathrm{a}}$ & 1.38 & $37.14^{\mathrm{a}}$ & $6.57^{\mathrm{a}}$ & $3.64^{\mathrm{a}}$ \\
\hline $25 \times 25$ & $22.17^{\mathrm{b}}$ & 1.15 & $20.18^{b}$ & $5.82^{\mathrm{b}}$ & $3.50^{\mathrm{b}}$ \\
\hline Significance & $*$ & N.S & * & * & * \\
\hline SEM & 8.40 & 0.42 & 4.10 & 0.12 & 0.10 \\
\hline \multicolumn{6}{|c|}{ Poultry manure (tha $\left.{ }^{-1}\right)$} \\
\hline 0 & $20.36^{c}$ & 1.42 & $18.42^{\mathrm{b}}$ & $4.12^{c}$ & $1.55^{\mathrm{c}}$ \\
\hline 5 & $28.21^{\mathrm{b}}$ & 1.45 & $22.14^{\mathrm{b}}$ & $5.14^{\mathrm{b}}$ & $2.67^{\mathrm{b}}$ \\
\hline 10 & $40.32^{a}$ & 1.48 & $35.47^{\mathrm{a}}$ & $6.68^{\mathrm{a}}$ & $3.42^{\mathrm{a}}$ \\
\hline 15 & $40.16^{\mathrm{a}}$ & 1.57 & $37.16^{\mathrm{a}}$ & $6.72^{\mathrm{a}}$ & $3.45^{\mathrm{a}}$ \\
\hline Significance & ${ }^{*}$ & N.S & ${ }^{*}$ & ${ }^{*}$ & * \\
\hline SEM & 7.40 & 0.42 & 4.10 & 0.12 & 0.10 \\
\hline$P^{*} S$ & N.S & N.S & N.S & N.S & N.S \\
\hline
\end{tabular}

Means followed by the same letter in a column are not significantly different at $5 \%$ level of probability. $\quad$ *Significant at $5 \%$ level of probability.

Table 7. Effect of poultry manure and spacing on nutrient uptake of onion bulb

\begin{tabular}{|c|c|c|c|c|c|}
\hline $20 \times 20$ & $85.48^{\mathrm{a}}$ & 7.25 & $81.24^{\mathrm{a}}$ & 18.19 & 6.78 \\
\hline $25 \times 25$ & $68.16^{\mathrm{b}}$ & 6.17 & $60.31^{\mathrm{b}}$ & 15.22 & 5.40 \\
\hline Significance & ${ }^{*}$ & N.S & ${ }^{*}$ & N.S & N.S \\
\hline SEM & 12.10 & 1.20 & 10.15 & 3.48 & 2.10 \\
\hline \multicolumn{6}{|c|}{ Poultry manure $\left(\right.$ kgha $\left.^{-1}\right)$} \\
\hline 0 & $48.77^{c}$ & 5.10 & $42.14^{c}$ & $10.12^{\mathrm{b}}$ & 5.15 \\
\hline 5 & $74.63^{\mathrm{b}}$ & 5.13 & $65.69^{\mathrm{b}}$ & $16.21^{2}$ & 5.18 \\
\hline 10 & $92.42^{a}$ & 6.39 & $84.15^{\mathrm{a}}$ & $17.10^{2}$ & 6.94 \\
\hline 15 & $98.69^{\mathrm{a}}$ & 6.27 & $87.43^{a}$ & $18.17^{a}$ & 7.17 \\
\hline Significance & * & N.S & ${ }^{*}$ & ${ }^{*}$ & N.S \\
\hline SEM & 12.10 & 1.28 & 10.15 & 3.48 & 2.10 \\
\hline \multicolumn{6}{|l|}{ Interaction } \\
\hline $\mathrm{F}^{*} \mathrm{~S}$ & N.S & N.S & N.S & N.S & N.S \\
\hline
\end{tabular}

Means followed by the same letter in a column are not significantly different at $5 \%$ level of probability.

${ }^{*}$ Significant at $5 \%$ level of probability.

Table 8. Effect of poultry manure rates and spacing on the proximate composition of onion leaves

\begin{tabular}{|c|c|c|c|c|c|}
\hline & Moisture content (\%) & Ash (\%) & Protein (\%) & Crude fibre (\%) & Dry matter $(\%)$ \\
\hline Spacing $(\mathrm{cm}) 15 \times 20$ & $87.62^{a}$ & $1.16^{a}$ & $3.44^{\mathrm{a}}$ & $1.16^{a}$ & $12.38^{a}$ \\
\hline $20 \times 20$ & $87.61^{a}$ & $1.21^{\mathrm{a}}$ & $3.47^{\mathrm{a}}$ & $1.18^{\mathrm{a}}$ & $12.39^{\mathrm{a}}$ \\
\hline $25 \times 25$ & $87.57^{a}$ & $1.23^{\mathrm{a}}$ & $3.48^{\mathrm{a}}$ & $1.19^{a}$ & $12.43^{\mathrm{a}}$ \\
\hline SEM & 0.24 & 0.04 & 0.03 & 0.03 & 0.23 \\
\hline \multicolumn{6}{|l|}{ Poultry manure $\left(t \mathrm{ha}^{-1}\right)$} \\
\hline 0 & $87.28^{b}$ & $1.06^{\mathrm{a}}$ & $3.42^{\mathrm{a}}$ & $1.01^{\mathrm{c}}$ & $12.72^{\mathrm{a}}$ \\
\hline 5 & $87.21^{\mathrm{b}}$ & $1.16^{\mathrm{a}}$ & $3.44^{\mathrm{a}}$ & $1.12^{\mathrm{b}}$ & $12.79^{\mathrm{a}}$ \\
\hline 10 & $87.65^{\mathrm{ab}}$ & $1.18^{\mathrm{a}}$ & $3.47^{\mathrm{a}}$ & $1.18^{\mathrm{a}}$ & $12.35^{\mathrm{ab}}$ \\
\hline 15 & $88.32^{\mathrm{a}}$ & $1.23^{\mathrm{a}}$ & $3.48^{\mathrm{a}}$ & $1.19^{\mathrm{a}}$ & $11.88^{b}$ \\
\hline SEM & 0.30 & 0.05 & 0.07 & 0.05 & 0.27 \\
\hline
\end{tabular}

Means followed by the same letter in a column are not significantly different at $5 \%$ level of probability using DMRT.

Table 9. Effect of poultry manure rates and spacing on the proximate composition of onion bulbs

\begin{tabular}{|c|c|c|c|c|c|c|}
\hline & $\begin{array}{c}\text { Moisture } \\
\text { content }(\%)\end{array}$ & $\operatorname{Ash}(\%)$ & Protein (\%) & Crude fibre (\%) & Crude fat $(\%)$ & $\begin{array}{c}\text { Dry matter } \\
(\%)\end{array}$ \\
\hline Spacing $(\mathrm{cm}) 15 \times 20$ & $87.67^{a}$ & $1.56^{\mathrm{a}}$ & $3.28^{a}$ & $1.18^{\mathrm{a}}$ & $0.80^{\mathrm{a}}$ & $12.33^{\mathrm{a}}$ \\
\hline $20 \times 20$ & $87.56^{\mathrm{a}}$ & $1.63^{\mathrm{a}}$ & $3.33^{\mathrm{a}}$ & $1.15^{\mathrm{a}}$ & $0.82^{\mathrm{a}}$ & $12.44^{\mathrm{a}}$ \\
\hline $25 \times 25$ & $87.71^{\mathrm{a}}$ & $1.65^{\mathrm{a}}$ & $3.38^{\mathrm{a}}$ & $1.21^{\mathrm{a}}$ & $0.82^{\mathrm{a}}$ & $12.29^{\mathrm{a}}$ \\
\hline SEM & 0.23 & 0.12 & 0.19 & 0.04 & 0.05 & 0.27 \\
\hline \multicolumn{7}{|l|}{ Poultry manure $\left(t \mathrm{ha}^{-1}\right)$} \\
\hline 0 & $85.35^{b}$ & $1.49^{\mathrm{a}}$ & $3.12^{\mathrm{b}}$ & $0.94^{\mathrm{b}}$ & $0.80^{\mathrm{a}}$ & $14.65^{\mathrm{a}}$ \\
\hline 5 & $85.32^{b}$ & $1.54^{\mathrm{a}}$ & $3.15^{b}$ & $1.15^{\mathrm{a}}$ & $0.82^{\mathrm{a}}$ & $14.68^{\mathrm{a}}$ \\
\hline 10 & $86.71^{\mathrm{a}}$ & $1.61^{\mathrm{a}}$ & $3.36^{\mathrm{a}}$ & $1.18^{\mathrm{a}}$ & $0.85^{\mathrm{a}}$ & $13.29^{\mathrm{b}}$ \\
\hline 15 & $86.95^{\mathrm{a}}$ & $1.63^{\mathrm{a}}$ & $3.39^{\mathrm{a}}$ & $1.21^{\mathrm{a}}$ & $0.85^{\mathrm{a}}$ & $13.05^{b}$ \\
\hline SEM & 0.29 & 0.18 & 0.20 & 0.12 & 0.06 & 0.32 \\
\hline
\end{tabular}

Means followed by the same letter in a column are not significantly different at $5 \%$ level of probability using DMRT. 


\section{Discussion}

The significant increase in crop growth rate, leaf thickness and harvest index as observed with the wider spacing of $25 \mathrm{~cm} \times 25 \mathrm{~cm}$ when compared with the narrower spacing of $15 \mathrm{~cm} \times 20 \mathrm{~cm}$ could be due to the fact that the plants in that environment had less competition for nutrient and other growth resources as opposed to the closer spacing of $15 \mathrm{~cm} \times 20 \mathrm{~cm}$ which resulted in competition for growth resources. The present results are supported by the findings of Maurya et al. (2013) who recorded maximum values at the widely spaced plant and minimum values in the narrowly spaced plants. This also corroborates the findings of Arisha et al. (2003) that wider spacing enhances vegetative growth of plants through the development of more thick leaves thereby enhancing these parameters. The superiority expressed by the wider spacing over the closely spaced was in accordance with the findings of Colla et al. (2002) on onion. In addition Jeyathilake et al. (2006) confirmed that closer spacing results in the growth of less vigorous plant when compared with wider spacing. The results of onion spacing experiment reported by Khan et al. (2002) showed that not only were the leaves of onion more from wider spacing but they had thicker and larger leaves. This was probably accounted for by a relatively reduced competition among plants per unit area for available soil nutrients in the lower density plots than the higher density ones.

The highest values recorded for all the vegetative and reproductive characters were obtained from 10 and $15 \mathrm{t} \mathrm{ha}^{-1}$ rate of the poultry manure throughout the period of study and this could be due to high rate of nutrient released from the manure. One of the methods through which plants would display its potential genetic capacity is by supplying the plants with adequate amount and types of fertilizer at the right time (Olaniyi, 2006). This positive response to increase in the level of manure application could probably be linked to the fact that onion plants have superficial root system that is rarely branched and lack root hair which has resulted in its high demand for nutrients. The main factor responsible for the increase in measured parameters as a result of increase in manure application could be due to increase availability of total assimilates for distribution to the bulb and shoot and in addition dry matter increased. The lowest values recorded from the control suggests that tropical soils are low in inherent plant nutrients which are required for plant growth and high yield, and thus necessitate the need to supplement the amount of nutrient in the soil to meet crop requirements Khushk et al. (1992). These results confirmed the report of Awodun (2007) that there is a significant influence on the growth and yield of Telfaria by application of fertilizers. This result is also in consonance with the findings of Aliyu and Kuchinda (2002) who stated that nitrogen which is a constituent of organic manure enhances physiological activities in crops; thereby improving the synthesis of photo-assimilates and a general increase in vegetative growth of plants. In contrast to growth parameters, significantly highest shoot and total dry and fresh yield was recorded with $20 \mathrm{~cm} \times 20 \mathrm{~cm}$ and 15 $\mathrm{cm} \times 20 \mathrm{~cm}$ and the least yield recorded for the widest spacing of $25 \mathrm{~cm} \times 25 \mathrm{~cm}$, this result could be attributed to greater crop biomass found with the narrower spacing as supported by Tijani-Eniola et al. (2003). The nutrient uptake was highest with $20 \mathrm{~cm} \times 20 \mathrm{~cm}$ plant spacing followed by $15 \mathrm{~cm} \times 20 \mathrm{~cm}$ and then $25 \mathrm{~cm} \times 25 \mathrm{~cm}$ spacing for all the treatments. This probably indicated that plants at this spacing might have experienced better soil conditions to enable them absorb these nutrients effectively, this result could also be attributed to the previous report of Tijani-Eniola et al. (2003). Wider spacing reduced yield due to total reduction in plants per hectare and consequently spacing may not be fully utilized. These results are evidently in accordance with that of Tijani-Eniola et al. (2003). The significant increase in $\mathrm{N}, \mathrm{K}$ and $\mathrm{Ca}$ contents of leaves and bulbs with increase in manure application could be explained by the work of Adekiya and Agbede (2009) who reported that all levels of poultry manure alone and NPK fertilizer + poultry manure increased leaf $\mathrm{N}, \mathrm{P}, \mathrm{K}, \mathrm{Ca}$ and $\mathrm{Mg}$ amounts significantly and the concentration of nutrients increased with the amount of poultry manure up to $40 \mathrm{t}$ ha. This indicate that increasing fertilizer rates affected levels of nutrients in leaf and bulb tissues and this is also in agreement with the work of Boyhan et al. (2007) who reported that a single fertilizer rate did not increase yield and nutrient content of onion, but increase in doses of $\mathrm{N}, \mathrm{P}$ and $\mathrm{K}$ affected levels of some nutrients in leaf and bulb tissue of onion. The lack of significant response in P content to increase in manure rates could be attributed to the presence of adequate amounts of available $\mathrm{P}$ in the soil (18.90 $\mathrm{mg} \mathrm{kg}^{-1}$ ).

Despite that spacing did not have a significant effect on the proximate composition of leaves and bulb of onion, the increase in the moisture content, crude fibre and crude protein as a result of increase in manure levels indicated that manure improved the quality of crops.

\section{Conclusions}

Based on the findings of the present study, growth of onion was enhanced at $20 \mathrm{~cm} \times 20 \mathrm{~cm}$ and $25 \mathrm{~cm} \times 25 \mathrm{~cm}$ compared to $15 \mathrm{~cm} \times 20 \mathrm{~cm}$ spacing. However, higher total fresh and dry yield of onion bulbs and shoots were obtained at $15 \mathrm{~cm} \times 20 \mathrm{~cm}$ which was not significantly different from $20 \mathrm{~cm} \times 20 \mathrm{~cm}$. Uptakes of $\mathrm{N}, \mathrm{K}$ and $\mathrm{Ca}$ increased with decrease in plant spacing and were higher at $15 \mathrm{~cm} \times 20 \mathrm{~cm}$ and $20 \mathrm{~cm} \times 20 \mathrm{~cm}$ spacing and at $10 \mathrm{tha}^{-1}$ and $15 \mathrm{t} \mathrm{ha}^{-1}$ poultry manure application.

\section{Acknowledgements}

This work was in part supported by Step B World Bank assisted project.

\section{References}

Adekiya AO, Agbede TM (2009). Growth and yield of tomato (Lycopersicon esculentum Mill) as influenced by poultry manure and NPK fertilizer.Journal of Food and Agriculture 21(1):10-20.

Adenawoola AR, Adejoro SA (2005). Residual effects of poultry manure and NPK fertilizer residues on soil nutrient and performance of Jute (Corchorus olitoriusL.). Nigerian Journal of soil science 15:133-135.

Aisha AH, RizkFA, Shaheen AM, Abdel Mouty MM (2007). Onion plant 
growth, bulbs yield and its physical and chemical properties as affected by organic and natural fertilizers. Research Journal of Agricultural and Biological Sciences 3(5):380-388.

Akinfasoye JA, Akanbi WB (2005). Effect of organic fertilizer and spacing on growth and yield of celosia (Celosia argentea L.). Proceedings 23rd HORTSON Conference, Port Harcourt September.

Aliyu L, Kuchinda NC ( 2002). Analysis of the chemical composition of some organic manures and their effect on the yield and composition of pepper (Capsium annum L). Crop Research 23(2):362-368.

Aliyu U, Dikko AU, Magaji MD, Singh A (2008). Nitrogen and intrarow spacing effect on onion (Allium cepa L.). Journal of Plant Science 3:188193.

Arisha HME, Gad AA, Younes SE (2003). Response of some pepper cultivars to organic and mineral nitrogen fertilizer under sandy soil conditions. Zagazig Journal of Agricultural Research 30:1875-1899.

Awodun MA (2007). Effect of poultry manure on growth, yield and nutrient content of fluted pumpkin (Telfaira occidentalis Hooks F). Asian Journal of Agricultural Research 1:67-73.

Baloch AW, Soomro AM, Javed MA, Ahmed M, Bughio HR, Bughio MS, Mastoi NN (2002). Optimum plant density for high yield in rice (Oryzasativa L.). Asian Journal of Plant Science 1:25-27.

Barker AV, Bryson GM (2006). Comparison of composts with low or high nutrient status for growth of plants in containers. Communications in Soil Science and Plant Analysis 37:1303-1319.

Boyhan GE, Torrance RL, Hill CR (2007). Effects of nitrogen, phosphorus, and potassium rates and fertilizer sources on yield and leaf nutrient status of short-day onions. HortScience 42:653-660.

Bray RH, Kurtz LT (1945). Determination of total organic and available forms of P in soils. Soil Science 59:39-45.

Brewster JL, Butler HA (1994). Effects of nitrogen supply on bulb development in onions(Allium cepa L.).Journal of Experimental Botany 40:1155-1162.

Chen SH, Hammond LL (1988). Agronomic evaluation of partially acidulated phosphate rocks in the tropics. IFDC's experience. IFDC 7:10.

Colla G, Mitchell J, Poudel D, Temple S (2002). Changes of tomatoes yield and fruit elemental composition in conventional, low input, and organic systems. Journal of Sustainable Agriculture 20(2):53-67.

Ehigiator JO (1998). Farmyard manure needs for its adoption as an alternative to chemical fertilizers uses in Nigeria. Nigerian Journal of Horticultural Science 3:19-24.

Hussaini MA, Amans EB, Ramalan AA (2000). Yield bulb size distribution and storability of onion (Allium cepa $\mathrm{L}$.) under Different levels of $\mathrm{N}$ fertilization and irrigation regime. Tropical Agriculture (Trinidad) $77(3): 145-49$.

Jackson ML (1969). Soil Chemical Analysis. Constable and Co Ltd, London, U.K.
Jeyathilake PKS, Reddy IP, Srihari D, Reddy KR (2006). Productivity and soil fertility status as influenced integrated use of N-Fixing Biofertilizers, organic manures and inorganic fertilizers in onion. Journal of Agricultural Science 2(1):46-58.

Khan HM, Igbal AG, Waseem K (2002). Effect of various plant spacing and different level of fertilizers on the growth and yield of onion (Allium cepa L.) Journal of Biological Sciences 2:545-547.

Khushk AM, Miano NM, Ansari AH (1992). Influence of inter and intra raw spacing on the yield and yield components of onion (Allium cepa $\mathrm{L}$.). Horticultural Abstracts 62:230-237.

Lombin LG, Adepelu JA, Ayotade BO (1991). Organic fertilizer in the Nigeria Agriculture. Proceeding of National Fertilizer Seminar, Kaduna, Nigeria. March 26-27 pp 5-13.

Maurya RP, Bailey JA, Chandler JSA (2013). Impact of plant spacing and picking interval on the growth, fruit quality and yield of okra. American Journal of Agriculture and Forestry 1(4):48-54.

Murphy J, Riley JP (1962). Modified single solution methods for determination of phosphorus in natural water. Analytical Chemistry Acta 27:31-36.

Mortley DG, Rhoden EG, Khan VA (1992). Plant spacing influences yield of vegetable amaranth. Acta Horticulturae 318:217-224.

NIHORT (1986). Advances in fruit and vegetable research at the National Horticultural Research Institute, Ibadan, Nigeria (NIHORT) (1976 1986). A commemorative publication to mark the 10th anniversary of the institute.

Olaniyan AA, Fagbayide JA, Kolade JA (2002). Nursery performances of citrus scion varieties on commercial rootstocks in Ibadan, south western Nigeria. Nigerian Journal of Horticultural Science 8(1):12-19.

Olaniyi JO (2006). Influence of Nitrogen and Phosphorus fertilizers on seed yield and quality of Egusi melon (Citrullus lanatus (Thunb) Mansf) in Ogbomoso, Southwestern Nigeria. PhD Thesis, University of Ibadan, pp 57-155.

Pandey OP, Srivastava BK, Singh MP (1996). Effect of spacing and fertility levels on the growth, yield and economics of tomato hybrids. Vegetable Science 23:9-15.

Soumare M, Tack FGM, Verloo MG (2003) Characterisation of Malian and Belgian solid waste composts with respect to fertility and suitability for land application. Waste Management 23 (6):517-522.

Tijani-Eniola H, Nolaeyo NU, Aiyelari OP, Nwagwu, FA (2003). Influence of crop density and delayed weeding on the performance of soybean (Glycine max. (L) Merrill). Nigerian Journal of Horticultural Science 8:53-60.

Walkley J, Black JP (1962). A critical examination of a rapid method for determining organic carbon in soils. Effects of variation in digestion conditions and of organic carbon constituents. Soil Science 63:251-263.

William CN, TO, Peregine WTH (1991). Vegetable production in the tropics Longman Scientific Technical Group. 